\title{
PARTIALLY RELAXED COCOERCIVE VARIATIONAL INEQUALITIES AND AUXILIARY PROBLEM PRINCIPLE
}

\author{
RAM U. VERMA
}

Received 30 May 2003 and in revised form 25 November 2003

Let $T: K \rightarrow H$ be a mapping from a nonempty closed convex subset $K$ of a finitedimensional Hilbert space $H$ into $H$. Let $f: K \rightarrow \mathbb{R}$ be proper, convex, and lower semicontinuous on $K$ and let $h: K \rightarrow \mathbb{R}$ be continuously Frećhet-differentiable on $K$ with $h^{\prime}$ (gradient of $h$ ), $\alpha$-strongly monotone, and $\beta$-Lipschitz continuous on $K$. Then the sequence $\left\{x^{k}\right\}$ generated by the general auxiliary problem principle converges to a solution $x^{*}$ of the variational inequality problem (VIP) described as follows: find an element $x^{*} \in K$ such that $\left\langle T\left(x^{*}\right), x-x^{*}\right\rangle+f(x)-f\left(x^{*}\right) \geq 0$ for all $x \in K$.

\section{Introduction}

The class of partially relaxed monotone variational inequalities is more general than the widely well-explored classes of strongly monotone as well as cocoercive variational inequalities in different space settings. As far as the solvability of this class of variational inequalities is concerned, in most of the cases, either projection or projection-type methods have been applied to finite-dimensional settings, because most of the nice applications happen to be in $\mathbb{R}^{n}$. In this paper, based on the generalized auxiliary problem principle, we plan to present the approximation-solvability of variational inequality problems (VIPs) involving partially relaxed cocoercive mappings, where the convergence analysis is more involved even in finite-dimensional settings than projection-type methods. As Frećhet-differentiable functions play a pivotal role in developing a general framework for the auxiliary problem principle, these investigations are new and in certain cases complement the work of El Farouq [7], Verma [21], and others. For more details on variational method, we recommend $[1,2,3,4,5,6,7,8,9,10,11,12,13,14,15,16,17,18,19,20$, $21,22]$.

Let $H$ be a finite-dimensional Hilbert space with the inner product $\langle\cdot, \cdot\rangle$ and the norm $\|\cdot\|$ on $H$. We consider the VIP as follows: determine an element $x^{*} \in K$ such that

$$
\left\langle T\left(x^{*}\right), x-x^{*}\right\rangle+f(x)-f\left(x^{*}\right) \geq 0, \quad \forall x \in K,
$$

Copyright (C) 2004 Hindawi Publishing Corporation Journal of Applied Mathematics and Stochastic Analysis 2004:2 (2004) 143-148 2000 Mathematics Subject Classification: 49J40, 65B05, 47H20

URL: http://dx.doi.org/10.1155/S1048953304305010 
where $K$ is a nonempty closed convex subset of $H$ and $f: K \rightarrow \mathbb{R}$ is a function on $K$. For $f \equiv 0$ in (1.1), it reduces to the problem: find an element $x^{*} \in K$ such that

$$
\left\langle T\left(x^{*}\right), x-x^{*}\right\rangle \geq 0, \quad \forall x \in K \text {. }
$$

We need now to define and in some cases to upgrade the existing notions in the literature. A mapping $T: K \rightarrow H$ is said to be monotone if

$$
\langle T(x)-T(y), x-y\rangle \geq 0, \quad \forall x, y \in K
$$

The mapping $T$ is $\alpha$-strongly monotone if there exists a constant $\alpha>0$ such that

$$
\langle T(x)-T(y), x-y\rangle \geq \alpha\|x-y\|^{2}, \quad \forall x, y \in K .
$$

The mapping $T$ is $\gamma$-cocoercive if there exists a constant $\gamma>0$ such that

$$
\langle T(x)-T(y), x-y\rangle \geq \gamma\|T(x)-T(y)\|^{2}, \quad \forall x, y \in K .
$$

A mapping $T: K \rightarrow H$ is said to be pseudomonotone if

$$
\langle T(y), x-y\rangle \geq 0 \Longrightarrow\langle T(x), x-y\rangle \geq 0, \quad \forall x, y \in K \text {. }
$$

$T: K \rightarrow H$ is $b$-strongly pseudomonotone if

$$
\langle T(y), x-y\rangle \geq 0 \Longrightarrow\langle T(x), x-y\rangle \geq b\|x-y\|^{2}, \quad \forall x, y \in K
$$

$T$ is $c$-pseudococoercive if there exists a constant $c>0$ such that

$$
\langle T(y), x-y\rangle \geq 0 \Longrightarrow\langle T(x), x-y\rangle \geq c\|T(x)-T(y)\|^{2}, \quad \forall x, y \in K .
$$

$T$ is quasimonotone if

$$
\langle T(y), x-y\rangle>0 \Longrightarrow\langle T(x), x-y\rangle \geq 0, \quad \forall x, y \in K
$$

$T$ is $L$-relaxed monotone (also referred to as weakly monotone) if there exists a constant $L>0$ such that

$$
\langle T(x)-T(y), x-y\rangle \geq(-L)\|x-y\|^{2}, \quad \forall x, y \in K
$$

$T$ is hemicontinuous if, for all $x, y, w \in K$, the function

$$
t \in[0,1] \longrightarrow\langle T[y+t(x-y)], w\rangle
$$

is continuous. $T$ is $\gamma$-partially relaxed monotone if there exists a constant $\gamma>0$ such that

$$
\langle T(x)-T(y), z-y\rangle \geq(-\gamma)\|z-x\|^{2}, \quad \forall x, y, z \in K .
$$


$T$ is $\gamma$-partially relaxed pseudomonotone if there exists a constant $\gamma>0$ such that

$$
\langle T(y), z-y\rangle \geq 0 \Longrightarrow\langle T(x), z-y\rangle \geq(-\gamma)\|z-x\|^{2}, \quad \forall x, y, z \in K .
$$

$T$ is said to be $\gamma$-r -partially relaxed cocoercive if there exist constants $\gamma, r>0$ such that

$$
\langle T(x)-T(y), z-y\rangle \geq(-\gamma)\|z-x\|^{2}+r\|T(x)-T(y)\|^{2} .
$$

This implies that $T$ is $\gamma$-partially relaxed monotone. $T$ is said to be $\gamma$ - $r$-partially relaxed pseudococoercive if

$$
\langle T(y), z-y\rangle \geq 0 \Longrightarrow\langle T(x), z-y\rangle \geq-\gamma\|z-x\|^{2}+r\|T(x)-T(y)\|^{2} .
$$

\section{Some auxiliary results}

In this section, we recall some auxiliary results crucial to the approximation-solvability of the VIP (1.1). Let $h: H \rightarrow \mathbb{R}$ be a continuously Fréchet-differentiable mapping on a Hilbert space $H$. It follows that $h^{\prime}(x) \in L(H, \mathbb{R})$, the space of all bounded linear operators from $H$ into $\mathbb{R}$. From now on, we will denote the real number $h^{\prime}(x)(y)$ by $\left\langle h^{\prime}(x), y\right\rangle$ for $x, y \in H$. The following result is a modified version of [2, Lemma 4.1].

Lemma 2.1. Let $H$ be a Hilbert space and $K$ a nonempty convex subset of $H$. Suppose that $h^{\prime}$, the gradient of $h: K \rightarrow \mathbb{R}$, is $\alpha$-strongly monotone on $K$, where $h: K \rightarrow \mathbb{R}$ is a continuously Fréchet-differentiable mapping.

Then, for all $x, x^{*} \in K$,

$$
h(x)-h\left(x^{*}\right)-\left\langle h^{\prime}\left(x^{*}\right), x-x^{*}\right\rangle \geq\left(\frac{\alpha}{2}\right)\left\|x-x^{*}\right\|^{2}
$$

Lemma 2.2. Let $H$ be a Hilbert space and $K$ a nonempty convex subset of $H$. Suppose that $h^{\prime}$ (gradient of $h$ ) is $\beta$-Lipschitz continuous, where $h: K \rightarrow \mathbb{R}$ is continuously Fréchetdifferentiable. Then, for all $x, x^{*} \in K$, the following inequality holds:

$$
h(x)-h\left(x^{*}\right)-\left\langle h^{\prime}\left(x^{*}\right), x-x^{*}\right\rangle \leq\left(\frac{\beta}{2}\right)\left\|x-x^{*}\right\|^{2}
$$

\section{General auxiliary problem principle}

This section deals with a discussion of the approximation-solvability of the VIP (1.1) based on the general auxiliary problem principle.

Algorithm 3.1. For a given iterate $x^{k}$, determine $x^{k+1}$ such that, for $k \geq 0$,

$$
\left\langle\rho T\left(x^{k}\right)+h^{\prime}\left(x^{k+1}\right)-h^{\prime}\left(x^{k}\right), x-x^{k+1}\right\rangle+\rho\left[f(x)-f\left(x^{k+1}\right)\right] \geq 0, \quad \forall x \in K,
$$

where $K$ is a nonempty closed convex subset of $H$.

For $f \equiv 0$ in Algorithm 3.1, we obtain the following algorithm. 
Algorithm 3.2. For a given iterate $x^{k}$, determine $x^{k+1}$ such that, for $k \geq 0$,

$$
\left\langle T\left(x^{k}\right)+h^{\prime}\left(x^{k+1}\right)-h^{\prime}\left(x^{k}\right), x-x^{k+1}\right\rangle \geq 0, \quad \forall x \in K,
$$

where $K$ is a nonempty closed convex subset of $H$.

We are just about ready to present, based on Algorithm 3.1, the approximationsolvability of the VIP (1.1).

THEOREm 3.3. Let $T: K \rightarrow H$ be any $\gamma$-r-partially relaxed cocoercive mapping from a nonempty closed convex subset $K$ of a finite-dimensional Hilbert space $H$ into $H$. Let $f$ : $K \rightarrow \mathbb{R}$ be proper, convex, and lower semicontinuous on $K$, and let $h: K \rightarrow \mathbb{R}$ be continuously Fréchet-differentiable on $K$ with $h^{\prime}$ (gradient of $h$ ), $\alpha$-strongly monotone, and $\beta$-Lipschitz continuous.

If, in addition, $x^{*} \in K$ is a solution of the VIP (1.1), then

(i) $\left\|T\left(x^{k}\right)-T\left(x^{*}\right)\right\| \rightarrow 0$ as $k \rightarrow \infty$;

(ii) the sequence $\left\{x^{k}\right\}$ generated by Algorithm 3.1 converges to $x^{*}$ for

$$
0<\rho<\frac{\alpha}{2 \gamma}
$$

Proof. To show that the sequence $\left\{x^{k}\right\}$ converges to $x^{*}$, a solution of the VIP (1.1), we need to compute the estimates. We define a function $\Lambda^{*}$ by

$$
\Lambda^{*}(x):=h\left(x^{*}\right)-h(x)-\left\langle h^{\prime}(x), x^{*}-x\right\rangle .
$$

Then, on applying Lemma 2.1, we have

$$
\Lambda^{*}(x):=h\left(x^{*}\right)-h(x)-\left\langle h^{\prime}(x), x^{*}-x\right\rangle \geq\left(\frac{\alpha}{2}\right)\left\|x^{*}-x\right\|^{2}, \quad \text { for } x \in K,
$$

where $x^{*}$ is a solution of the VIP (1.1). It follows that

$$
\Lambda^{*}\left(x^{k+1}\right)=h\left(x^{*}\right)-h\left(x^{k+1}\right)-\left\langle h^{\prime}\left(x^{k+1}\right), x^{*}-x^{k+1}\right\rangle .
$$

Now, we can write

$$
\begin{aligned}
\Lambda^{*}\left(x^{k}\right)-\Lambda^{*}\left(x^{k+1}\right)= & h\left(x^{k+1}\right)-h\left(x^{k}\right)-\left\langle h^{\prime}\left(x^{k}\right), x^{k+1}-x^{k}\right\rangle \\
& +\left\langle h^{\prime}\left(x^{k+1}\right)-h^{\prime}\left(x^{k}\right), x^{*}-x^{k+1}\right\rangle \\
\geq & \left(\frac{\alpha}{2}\right)\left\|x^{k+1}-x^{k}\right\|^{2}+\left\langle h^{\prime}\left(x^{k+1}\right)-h^{\prime}\left(x^{k}\right), x^{*}-x^{k+1}\right\rangle \\
\geq & \left(\frac{\alpha}{2}\right)\left\|x^{k+1}-x^{k}\right\|^{2}+\rho\left\langle T\left(x^{k}\right), x^{k+1}-x^{*}\right\rangle \\
& +\rho\left(f\left(x^{k+1}\right)-f\left(x^{*}\right)\right)
\end{aligned}
$$

for $x=x^{*}$ in (3.1). 
If we replace $x$ by $x^{k+1}$ in (1.1), we obtain

$$
\left\langle\left(T\left(x^{*}\right), x^{k+1}-x^{*}\right)\right\rangle+f\left(x^{k+1}\right)-f\left(x^{*}\right) \geq 0 .
$$

Since $T$ is $\gamma$-r -partially relaxed cocoercive, it implies, in light of (3.8), that

$$
\begin{aligned}
\Lambda^{*}\left(x^{k}\right)-\Lambda^{*}\left(x^{k+1}\right) & \geq\left(\frac{\alpha}{2}\right)\left\|x^{k+1}-x^{k}\right\|^{2}+\rho\left\langle T\left(x^{k}\right)-T\left(x^{*}\right), x^{k+1}-x^{*}\right\rangle \\
& \geq\left[\frac{\alpha}{2}\right]\left\|x^{k+1}-x^{k}\right\|^{2}-\rho \gamma\left\|x^{k+1}-x^{k}\right\|^{2}+\rho r\left\|T\left(x^{k}\right)-T\left(x^{*}\right)\right\|^{2} \\
& =\left[\frac{\alpha}{2}-\rho \gamma\right]\left\|x^{k+1}-x^{k}\right\|^{2}+\rho r\left\|T\left(x^{k}\right)-T\left(x^{*}\right)\right\|^{2}, \quad \text { for } \rho<\frac{\alpha}{2 \gamma} .
\end{aligned}
$$

It follows from (3.9) that the sequence $\left\{\Lambda^{*}\left(x^{k}\right)\right\}$ is strictly decreasing except for $x^{k+1}=$ $x^{k}$, and in that situation, $x^{k}$ is a solution to (1.1). Since the difference of two consecutive terms of the sequence $\left\{\Lambda^{*}\left(x^{k}\right)\right\}$ tends to zero as $k \rightarrow \infty$, it implies that

$$
\left\|x^{k+1}-x^{k}\right\| \longrightarrow 0, \quad\left\|T\left(x^{k}\right)-T\left(x^{*}\right)\right\| \longrightarrow 0 \quad \text { as } k \longrightarrow \infty .
$$

On top of that, in light of (3.5), we have

$$
\left\|x^{*}-x^{k}\right\|^{2} \leq\left(\frac{2}{\alpha}\right) \Lambda^{*}\left(x^{k}\right)
$$

and so the sequence $\left\{x^{k}\right\}$ is bounded. Let $x^{\prime}$ be a cluster point of the sequence $\left\{x^{k}\right\}$, that is, there exists a subsequence $\left\{x^{k j}\right\}$ of the sequence $\left\{x^{k}\right\}$ such that $\left\{x^{k j}\right\}$ converges to $x^{\prime}$.

We replace $x^{*}$ by $x^{\prime}$ and define another function $\Lambda^{\prime}\left(x^{k}\right)$. Then the analysis is still similar to that of $\Lambda^{*}\left(x^{k}\right)$ and, as a result, the sequence $\left\{\Lambda^{\prime}\left(x^{k}\right)\right\}$ strictly decreases, and by Lemma 2.2, we have

$$
\Lambda^{\prime}\left(x^{k j}\right) \leq\left(\frac{\beta}{2}\right)\left\|x^{\prime}-x^{k j}\right\|^{2}
$$

Here, the sequence $\left\{\Lambda^{\prime}\left(x^{k j}\right)\right\} \rightarrow 0$. On the other hand, we have

$$
\Lambda^{\prime}\left(x^{k j}\right) \geq\left(\frac{\alpha}{2}\right)\left\|x^{k j}-x^{\prime}\right\|^{2}
$$

This implies that $x^{k j} \rightarrow x^{\prime}$, and hence the entire sequence converges to $x^{\prime}$. This completes the proof.

Theorem 3.4. Let $T: K \rightarrow H$ be a $\gamma$-r-partially relaxed cocoercive mapping from a nonempty closed convex subset $K$ of a finite-dimensional Hilbert space $H$ into $H$. Let $h: K \rightarrow \mathbb{R}$ be continuously Fréchet-differentiable on $K$ with $h^{\prime}$ (gradient of $h$ ), $\alpha$-strongly monotone, and $\beta$-Lipschitz continuous. If, in addition, $x^{*} \in K$ is a solution of the VIP (1.2), then the sequence $\left\{x^{k}\right\}$ generated by Algorithm 3.2 converges to $x^{*}$. 


\section{References}

[1] I. K. Argyros and R. U. Verma, On general auxiliary problem principle and nonlinear mixed variational inequalities, Nonlinear Funct. Anal. Appl. 6 (2001), no. 2, 247-256.

[2] - Generalized partial relaxed monotonicity and solvability of nonlinear variational inequalities, Panamer. Math. J. 12 (2002), no. 3, 85-104.

[3] G. Cohen, Auxiliary problem principle and decomposition of optimization problems, J. Optim. Theory Appl. 32 (1980), no. 3, 277-305.

[4] - Auxiliary problem principle extended to variational inequalities, J. Optim. Theory Appl. 59 (1988), no. 2, 325-333.

[5] J. Eckstein, Nonlinear proximal point algorithms using Bregman functions, with applications to convex programming, Math. Oper. Res. 18 (1993), no. 1, 202-226.

[6] J. Eckstein and D. P. Bertsekas, On the Douglas-Rachford splitting method and the proximal point algorithm for maximal monotone operators, Math. Programming 55 (1992), no. 3, 293-318.

[7] N. El Farouq, Pseudomonotone variational inequalities: convergence of proximal methods, J. Optim. Theory Appl. 109 (2001), no. 2, 311-326.

[8] , Pseudomonotone variational inequalities: convergence of the auxiliary problem method, J. Optim. Theory Appl. 111 (2001), no. 2, 305-326.

[9] S. Karamardian, Complementarity problems over cones with monotone and pseudomonotone maps, J. Optimization Theory Appl. 18 (1976), no. 4, 445-454.

[10] S. Karamardian and S. Schaible, Seven kinds of monotone maps, J. Optim. Theory Appl. 66 (1990), no. 1, 37-46.

[11] B. Martinet, Régularisation d'inéquations variationnelles par approximations successives, Rev. Française Informat. Recherche Opérationnelle 4 (1970), 154-158 (French).

[12] Z. Naniewicz and P. D. Panagiotopoulos, Mathematical Theory of Hemivariational Inequalities and Applications, Monographs and Textbooks in Pure and Applied Mathematics, vol. 188, Marcel Dekker, New York, 1995.

[13] R. T. Rockafellar, Augmented Lagrangians and applications of the proximal point algorithm in convex programming, Math. Oper. Res. 1 (1976), no. 2, 97-116.

[14] Monotone operators and the proximal point algorithm, SIAM J. Control Optimization 14 (1976), no. 5, 877-898.

[15] R. U. Verma, Nonlinear variational and constrained hemivariational inequalities involving relaxed operators, Z. Angew. Math. Mech. 77 (1997), no. 5, 387-391.

[16] _ Approximation-solvability of nonlinear variational inequalities involving partially relaxed monotone (PRM) mappings, Adv. Nonlinear Var. Inequal. 2 (1999), no. 2, 137-148.

[17] A class of projection-contraction methods applied to monotone variational inequalities, Appl. Math. Lett. 13 (2000), no. 8, 55-62.

[18] - Generalized multivalued implicit variational inequalities involving the Verma class of mappings, Math. Sci. Res. Hot-Line 5 (2001), no. 2, 57-64.

[19] - A new class of iterative algorithms for approximation-solvability of nonlinear variational inequalities, Comput. Math. Appl. 41 (2001), no. 3-4, 505-512.

[20] - Projection methods and a new system of cocoercive variational inequality problems, Int. J. Differ. Equ. Appl. 6 (2002), no. 4, 359-367.

[21] Nonlinear implicit variational inequalities involving partially relaxed pseudomonotone mappings, Comput. Math. Appl. 46 (2003), no. 10-11, 1703-1709.

[22] E. Zeidler, Nonlinear Functional Analysis and Its Applications. II/B, Springer-Verlag, New York, 1990.

Ram U. Verma: Department of Mathematics, The University of Toledo, Toledo, OH 43606, USA

E-mail address: rverma@pop3.utoledo.edu 


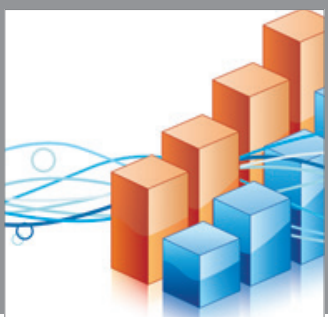

Advances in

Operations Research

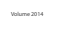

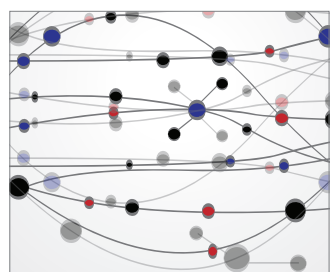

\section{The Scientific} World Journal
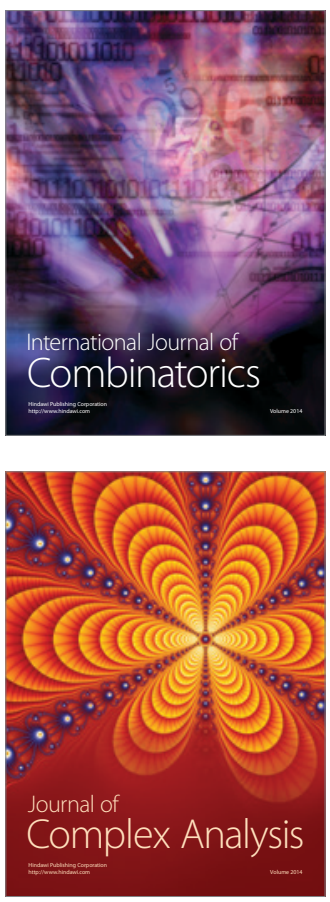

International Journal of

Mathematics and

Mathematical

Sciences
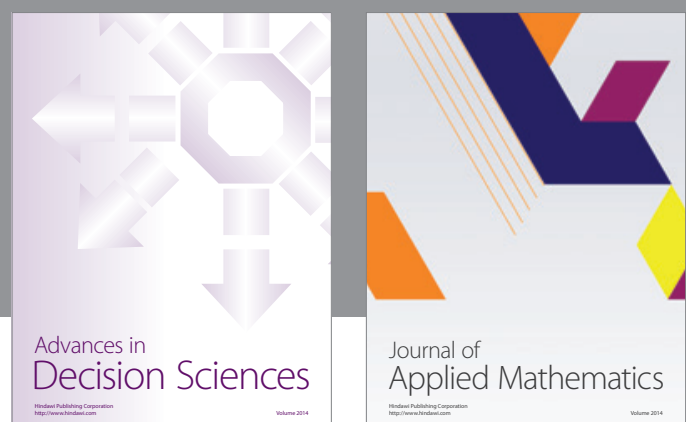

Journal of

Applied Mathematics
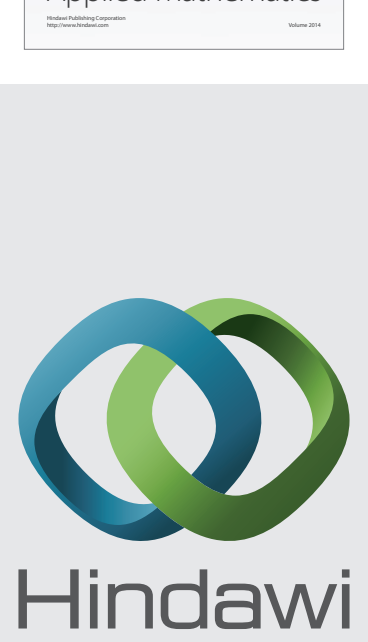

Submit your manuscripts at http://www.hindawi.com
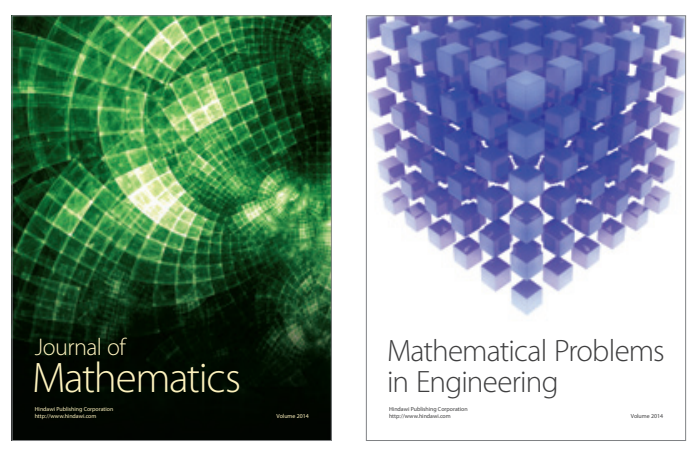

Mathematical Problems in Engineering
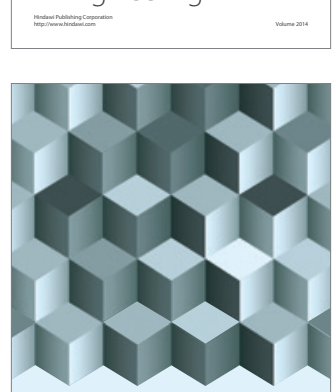

Journal of

Function Spaces
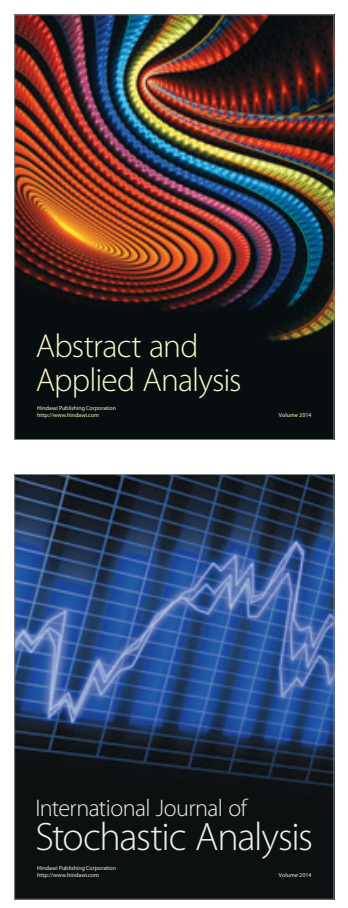

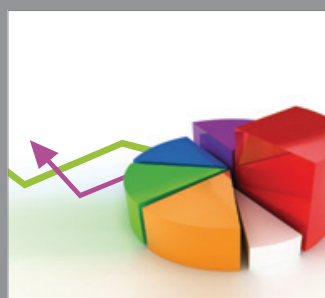

ournal of

Probability and Statistics

Promensencen
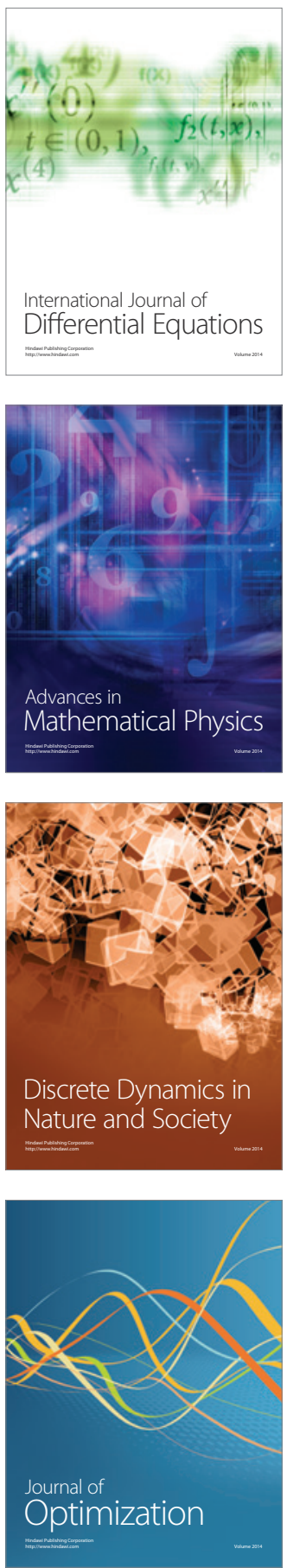